\title{
O enfermeiro na detecção precoce do câncer de mama no âmbito da atenção primária
}

\author{
The nurse in the early detection of breast cancer in primary health care \\ El enfermero en la detección precoz del cáncer de mama en la atención primaria
}

\author{
Ana Luiza Barreto Zapponi'; Florence Romijn Tocantins ${ }^{\text {II }}$; Octavio Muniz da Costa Vargens ${ }^{\text {III }}$
}

\begin{abstract}
RESUMO: Os objetivos foram identificar as ações desenvolvidas pelos enfermeiros na atenção à saúde da mulher e discutir a ação profissional do enfermeiro na detecção precoce do câncer de mama no âmbito da atenção primária. Trata-se de estudo descritivo com abordagem qualitativa fenomenológica, realizado no período de dezembro/2011 a novembro/2012. Os participantes foram 12 enfermeiros que desenvolvem ações voltadas para a saúde da mulher na atenção primária Os dados foram obtidos através de entrevistas semiestruturtadas gravadas e transcritas para posterior análise. As ações identificadas foram categorizadas tendo por fundamento o sentido em comum das ações dos enfermeiros. Constatou-se que o enfermeiro, na atenção primária, não tem como propósito da sua ação profissional a detecção precoce de anormalidades na mama, mas sim o cumprimento de ações preventivas voltadas principalmente para o período gravídico. Conclui-se que urge o redirecionamento das ações do enfermeiro visando a priorização da detecção de anormalidades na mama.

Palavras-Chave: Cuidados de enfermagem; atenção primária à saúde; diagnóstico precoce; câncer de mama.
\end{abstract}

ABSTRACT: The objectives were to identify the actions performed by nurses in caring for women and to discuss the nurses' professional action regarding the early detection of breast cancer in primary health care. This is an descriptive study with a phenomenological qualitative approach, carried out from December/2011 to November/2012. The participants were 12 nurses working with women's health in primary care. Data were collected through semi-structured interviews, which were recorded and transcribed for later analysis. The actions identified were categorized based on the common sense of nurses' actions. It was identified that nurses in primary health care, do not have the purpose in their professional action the early detection of abnormalities in the breast, but the implementation of preventive actions aimed mainly to the pregnancy period. It is concluded that is urgent to redirect of nurses' actions in order to prioritize the early detection of abnormalities in the breast. Keywords: Nursing care; primary health care; early diagnosis; breast neoplasms.

RESUMEN: Los objetivos fueron identificar las acciones de enfermeros en el cuidado a la salud de la mujer y discutir su acción profesional en relación a la detección precoz del cáncer de mama en la atención primaria de salud. Es un estudio descriptivo con abordaje cualitativo fenomenológico, realizado de diciembre/2011 a noviembre/2012. Participaron 12 enfermeros que trabajan con la salud de las mujeres en la atención primaria. Los datos fueron recolectados a través de entrevistas semiestructuradas, grabadas y transcritas para su análisis posterior. Las acciones identificadas fueron clasificadas por el sentido común de las acciones de enfermería. Fue identificado que las enfermeras en la atención primaria de salud, no tienen en su acción profesional el propósito de la detección temprana de anomalías en la mama, pero la implementación de acciones preventivas destinadas principalmente al embarazo. Se concluye que urge reorientar las acciones de enfermería con el fin de priorizar la detección temprana de anomalías mamárias.

Palabras Clave: Atención de enfermería; atención primaria de salud; diagnóstico precoz; neoplasias de la mama.

\section{INTRODUÇÃO}

O câncer de mama é a primeira causa de morte entre as mulheres ${ }^{1}$. Mundialmente, esta neoplasia é o mais comum entre as mulheres, abrangendo $23 \%$ dos cânceres femininos que são diagnosticados em mais de 1,1 milhões de mulheres a cada ano ${ }^{2,3}$. A mortalidade anual desta neoplasia é de mais de 411 mil mortes sendo responsável por mais de 1,6\% dos óbitos femininos por todas as causas, e o segundo tipo de câncer mais frequente no mundo ${ }^{4}$. No Brasil, o câncer de mama também é o mais incidente entre as mulheres, correspondendo por $22 \%$ dos casos novos a cada ano e com estimativa de 57.120 mil novos casos no ano de $2015^{5}$.

O câncer de mama é um problema de saúde pública no Brasil e um desafio que vem representando para o sistema de saúde no sentido de se garantir o acesso pleno e equilibrado da população ao diagnóstico e tratamento desta doença ${ }^{6}$. Sendo assim, o diagnóstico precoce deve-se fazer tão logo o processo da

\footnotetext{
'Enfermeira Obstétrica. Mestre em Enfermagem. Doutoranda em Enfermagem da Universidade do Estado do Rio de Janeiro. Brasil. E-mail: analuu@gmail.com. IIDoutora em Enfermagem. Professor Titular da Universidade Federal do Estado do Rio de Janeiro. Brasil. E-mail: florenceromijn@hotmail.com. IIIDoutor em Enfermagem. Professor Titular da Universidade do Estado do Rio de Janeiro. Brasil. E-mail: omcvargens@uol.com.br.
} 
doença seja detectável, no início de patogênese ${ }^{7}$. Para detecção precoce do câncer de mama, recomenda-se rastreamento por meio do exame clínico da mama; rastreamento por mamografia para as mulheres com idade entre 50 a 69 anos; exame clínico das mamas e mamografia anual a partir dos 35 anos para mulheres pertencentes a grupos populacionais com risco elevado de desenvolver câncer de mama e a garantia de acesso ao diagnóstico, tratamento e seguimento para todas as mulheres com alterações nos exames realizados ${ }^{8}$.

Nos últimos três anos, o Instituto Nacional do Câncer (INCA) e o Ministério da Saúde vêm ampliando a estratégia de alerta para esta neoplasia tanto às mulheres quanto para os profissionais de saúde ${ }^{5}$. Essa estratégia de comunicação preconiza que todas as mulheres devem conhecer os principais fatores de risco para o câncer de mama, a idade de maior risco de ocorrência da doença e seus mais frequentes sinais e sintomas. Também recomenda que as mulheres, ao identificarem tais sinais e sintomas procurem imediatamente um serviço de saúde para esclarecimento diagnóstico. Neste contexto, a atenção básica possibilita a participação ativa das mulheres perante a sua saúde na promoção e identificação precoce da doença.

A atenção básica caracteriza-se por um conjunto de ações de saúde, no âmbito individual e coletivo, que abrange a promoção e a proteção da saúde, a prevenção de agravos, o diagnóstico, o tratamento, a reabilitação, redução de danos e a manutenção da saúde com o objetivo de desenvolver uma atenção integral que impacte na situação de saúde e autonomia das pessoas e nos determinantes e condicionantes de saúde das coletividades ${ }^{9}$. Faz parte da atribuição profissional do enfermeiro realizar, na atenção primária a saúde, a assistência integral aos indivíduos e famílias em todas as fases de desenvolvimento humano ${ }^{9,10}$. Portanto, são de responsabilidade do enfermeiro, a partir do atendimento integral, estimular o empoderamento da mulher quanto sua saúde e seu corpo. Com este entendimento o profissional, nas consultas voltadas a saúde da mulher, deve ser capaz de detectar precocemente anormalidades na mama da mulher que possa ser eventualmente a fase inicial do câncer de mama.

Os elevados índices de incidência e mortalidade por câncer de mama no Brasil justificam a implantação de estratégias efetivas de controle dessa doença que incluam ações de promoção à saúde, prevenção e detecção precoce ${ }^{11}$. A Política Nacional de Atenção Integral à Saúde da Mulher reitera a afirmação anterior ao destacar que o câncer de mama é diagnosticado tardiamente em $60 \%$ dos casos e que mudar essa situação é um desafio necessário, já que o diagnóstico precoce aumenta significamente a perspectiva e a qualidade de vida das mulheres ${ }^{12}$.

A detecção precoce deve ser descoberta nesse nível de atenção para evitar a detecção no estádio avançado, uma vez que nesse estádio favorece a mortalidade por esta causa específica. Ainda, mesmo nesse nível de atenção a relação profissional-usuário de saúde se faz presente em condições que possibilitam um vínculo sem necessariamente a mulher estar doente (ou não), criando uma relação de corresponsabilidade e permitindo o protagonismo dessa mulher perante a sua saúde.

Sendo assim, os objetivos deste estudo foram: identificar as ações desenvolvidas pelos enfermeiros na atenção a saúde da mulher e discutir a ação profissional do enfermeiro na detecção precoce do câncer de mama no âmbito da atenção primária.

\section{Referencial Teórico-Metodológico}

A busca pela compreensão do significado da ação do enfermeiro na detecção precoce do câncer de mama no âmbito da atenção primária apoia-se no referencial teórico da fenomenologia de Alfred Schütz ${ }^{13-16}$.

A fenomenologia, de acordo com Husserl, visa a descrever as coisas nelas mesmas, tais como elas se manifestam à consciência. Visa desvelar aquilo que está presente naquilo que se mostra à consciência. Desse modo, não se caracteriza por ser um método com fins meramente práticos. Se propõe a uma compreensão e interpretação humana tal como ela é vivida e como se apresenta antes de se tornar objeto da percepção científica ${ }^{13}$. A fenomenologia social de Schütz tem como fundamento que toda ação que o sujeito desenvolve têm um sentido intencional e busca aí atender suas expectativas. Contudo este sentido e significado somente o próprio indivíduo pode desve$\operatorname{lar}^{14}$. Os motivos de cada sujeito são expressos em ações no mundo da vida natural. Quando os motivos deste sujeito são expressos em ações ao se dirigir ao outro e este do mesmo modo se reporta com uma ação, Schütz ${ }^{15}$ denomina de relação social.

Ao falar de relação social, Schütz ressalta que esta tem características próprias, cujas ações ocorrem de maneira consciente e intencional, possuindo um significado para o ator. Nesse sentido, o aspecto crucial da ação é o seu caráter intencional e de projeção, que tem a sua origem na consciência da pessoa e, como tal, toda e qualquer ação só pode ser compreendida mediante o significado que esta pessoa confere a sua ação - a interpretação do significado de uma ação só pode ocorrer pela subjetividade da própria pessoa ${ }^{16}$.

Para compreender o fenômeno estudado - o significado da ação profissional do enfermeiro na detecção precoce do câncer de mama no âmbito da atenção primária -, faz-se necessário voltar à consciência do profissional que realiza a assistência e o cuidado. De acordo com a fenomenologia de Schütz $z^{15}$, apreende-se o significado de uma ação através da apreensão dos motivos para. Estes se referem à finalidade de uma ação, ao resultado que se espera alcançar. Os mo- 
tivos para instigam a realização da ação e, portanto, estão dirigidos para o futuro. Assim, a meta consiste em descobrir os motivos que estão impulsionando a ação humana e, ainda assim, cada unidade de ação humana é só um corte que o observador extrai do contexto social total. Assim, quando o enfermeiro realiza suas ações, junto ao próximo, materializa a intencionalidade em forma dessas ações. É mediante a relação com o próximo que é possível estabelecer a assistência à saúde da mulher no âmbito da atenção primária, fazendo o movimento próprio do mundo profissional em que convivemos ${ }^{17}$.

Sob esta perspectiva, a ação do enfermeiro voltada para a saúde da mulher na atenção primária traz em si uma atitude reflexiva sobre a ação que tem em vista uma intencionalidade ao realizar ações profissionais direcionadas para determinado fim, os motivo para. Daí a importância de se buscar o significado desta ação por meio da fenomenologia social, a partir da análise das relações sociais entre sujeitos, entre o enfermeiro e a mulher atendida na atenção primária.

Desse modo, buscou-se em Schütz ${ }^{15}$ fundamentação para compreender o significado da ação do enfermeiro na atenção a saúde, possibilitando analisar a perspectiva da detecção precoce do câncer de mama na assistência a saúde da mulher na atenção primária a saúde.

\section{Método}

Trata-se de uma pesquisa qualitativa, apoiada na fenomenologia sociológica de Schütz ${ }^{15}$. $O$ estudo foi desenvolvido no município do Rio de Janeiro, tendo por referência as ações desenvolvidas nas unidades básicas de saúde - Centros Municipais de Saúde e Estratégias da Saúde da Família - e como tal, com ações previstas, de promoção, prevenção e recuperação, voltadas para a saúde da mulher.

Os sujeitos da pesquisa foram 12 enfermeiros que desenvolvem tais ações no contexto da atenção básica. Estes foram identificados por E1 a E12, de acordo com a letra e a inserção no processo da realização das entrevistas. A coleta de dados, realizada no período de dezembro de 2011 a fevereiro de 2012, ocorreu através de entrevistas fenomenológicas, gravadas em equipamento mp3 e transcritas na íntegra para posterior análise. $\mathrm{O}$ instrumento de coleta de dados foi um roteiro de entrevista constando questões sobre as atividades desenvolvidas no atendimento à mulher e uma questão fenomenológica: $\bigcirc$ que você tem em vista ao assistir a mulher na unidade básica de saúde?

A análise das informações e das entrevistas teve como fundamento o referencial teórico metodológico de Schütz ${ }^{15}$. As transcrições das entrevistas gravadas trouxeram as questões acerca das atividades desenvolvidas pelo enfermeiro no atendimento à mulher e as respostas quanto à questão fenomenológica. Nessa etapa do estudo, houve a leitura e a releitura dessas entrevistas com o intuito de identificar as atividades desenvolvidas pelos enfermeiros e agrupá-las por afinidade, procurando identificar as categorias que comportam a ação do enfermeiro além de identificar os motivos para dos mesmos.

Em atendimento às Diretrizes e Normas Regulamentadoras de Pesquisas Envolvendo Seres Humanos Resolução n 466/2012 do Conselho Nacional de Saúde, a pesquisa foi submetida à apreciação e aprovada pelo Comitê de Ética em Pesquisa da Secretaria Municipal de Saúde e Defesa Civil-RJ (CAAE:0036.0.313.314-11) e pelo Comitê de Ética em Pesquisa da Universidade Federal do Estado do Rio de Janeiro (UNIRIO). Todos os participantes assinaram Termo de Consentimento Livre e Esclarecido antes do início das entrevistas.

\section{Resultados e Discussão}

Após a leitura e a releitura exaustiva das entrevistas, as atividades desenvolvidas pelos enfermeiros que atendem as mulheres nas unidades básicas de saúde foram agrupadas por afinidade procurando identificar aquelas que comportam a ação do enfermeiro. Três atividades-ações emergiram das entrevistas sendo elas de encaminhamento, educativas e ações voltadas ao corpo físico.

Os encaminhamentos realizados pelo enfermeiro na atenção primária a saúde tem como fundamento a organização direta e/ou indireta no funcionamento da instituição. A relação estabelecida com outros profissionais de saúde se dá através da necessidade de resolver situações na qual o enfermeiro não tem habilitação ou não se sente apto para realizá-las. Já as ações educativas estão relacionadas com a educação em saúde em diferentes cenários, não tendo apenas a instituição como local para atividades de promoção a saúde. A ação do enfermeiro nesse contexto é estimular a participação da população para o cuidado com a saúde, reconhecendo o seu corpo. A terceira atividade-ação que emergiu das entrevistas foram ações voltadas ao corpo físico. Esta atividade-ação emergiu dos discursos de todos os entrevistados. A atenção do enfermeiro ao corpo físico da mulher é uma das principais ações profissionais realizadas por ele.

É... exame físico? Meço o fundo de útero, ausculto, é... (E1)

Tem aquelas ações de rotina [...] Que seria o exame físico, a coleta da história pregressa, medida de fundo uterino, ausculta de BCF, as prescrições dos medicamentos de rotina do ministério, a gente alcançou essa independência (E2)

Nota-se que as ações voltadas para o corpo feminino se concentram no período gravídico, e muitas dessas ações estão voltadas para o corpo físico da mul- 
her objetivamente e subjetivamente para o bem-estar do feto. Ainda com o foco no corpo físico da mulher, a ação profissional também consiste na prevenção de anormalidades no corpo, outro tema que emergiu nesta categoria:

Assim, o exame físico, detectar se ela tem algum problema, se ela tem edema... Porque a gente faz o exame físico também... (E1)

[...] fazer uma boa anamnese, orientá-la bem, para que ela tenha um parto seguro, uma gravidez mais segura, o bebê nascer saudável, ver esse binômio feliz...[...] diminuir a incidência dos problemas mais comuns, da morbimortalidade materna e infantil e que nasça uma criança saudável. (E2)

Depois que elas têm o neném a gente fala dos cuidados, quais as vacinas que têm que tomar, [...] do cuidado na amamentação. Tudo isso a gente faz nesse período [...] (E9)

O enfoque do enfermeiro ao orientar a mulher no atendimento consiste em evitar qualquer dano para a mulher e/ou para o recém-nascido, sendo também uma estratégica para ela se sentir segura em relação a questionamentos relacionados a sinais e sintomas de alguma anormalidade no cuidado com o seu corpo e/ ou com o recém-nascido.

E as orientações. Porque no certo, as orientações seriam a parte da enfermagem [...] (E1)

Após a leitura e a releitura exaustiva das entrevistas, observaram-se ações, que emergiram dos depoimentos as quais permitem afirmar que os enfermeiros que atendem as mulheres, nas unidades básicas de saúde, focalizam o corpo físico da mulher no período gravídico. Seu significado foi apreendido através da sua intencionalidade - motivo para, de acordo com Schütz $z^{15}$. A partir da organização e análise do conjunto dos motivos para emergiram ideias comuns, vivenciadas pelos sujeitos da ação, denominadas categorias concretas do vivido ${ }^{16}$. Esta análise permitiu o emergir da categoria concreta do vivido: evitar problemas de saúde da mulher. Ou seja, esse é o significado da ação profissional do enfermeiro na atenção à saúde da mulher na atenção primária.

Entende-se que no âmbito da atenção primária, a atuação profissional do enfermeiro abrange ações preventivas, de diagnóstico, tratamento e recuperação desse grupo da população no cenário da clínica ginecológica, pré-natal, parto e puerpério, climatério, planejamento familiar, doenças sexualmente transmissíveis, câncer do colo de útero e de mama, assim como o atendimento de outras necessidades identificadas a partir do perfil populacional das mulheres ${ }^{9,12}$.

Dessa forma, ao identificar como ação principal do enfermeiro aquelas voltadas para o corpo físico da mulher, cujo significado seja evitar problemas de saúde, compreende-se que nessas ações o profissional estabelece uma atitude que visa a identificar precoce- mente alterações corporais nos vários períodos de vida da mulher, atingindo assim as ações preventivas e de diagnóstico que os vários programas do Ministério da Saúde preconizam ${ }^{6,8,11,18,19}$.

No entanto, observa-se que a categoria concreta do vivido que emergiu da intencionalidade do enfermeiro que assiste as mulheres na atenção básica (evitar problemas) está presente no evitar problemas da mulher grávida, voltando sua ação profissional para a mulher que se encontra neste período específico da vida. Destaca-se, nessa perspectiva, que o enfermeiro ao assistir a mulher não tem como ação profissional a detecção precoce de anormalidades na mama. A ação do enfermeiro voltada para a mama da mulher, no contexto analisado, está direcionada para a amamentação do filho.

[...] realizar a prevenção e para ela se beneficiar mesmo, para evitar a mastite...(E6)

Assim, as medidas para evitar problemas estão voltadas para ações que prejudiquem o processo do aleitamento materno: a mama está inserida nas ações assistenciais para a relação mãe-bebê e não para a mulher cidadã.

Tendo em vista a importância da detecção precoce do câncer de mama, faz-se necessária a qualificação das equipes da atenção primária nessa função ${ }^{5}$. O rastreamento do câncer de mama deve acontecer na relação entre a captação das mulheres para a unidade básica de saúde, na atuação dos profissionais dessas unidades com as usuárias do sistema único de saúde e no diálogo participativo entre as Unidades Básicas de Saúde com a média e alta complexidade, colocando na prática a concepção da integralidade da assistência ${ }^{5,11}$. Desse modo, o cuidado à saúde deve ser permeado pelo acolhimento com escuta sensível de suas demandas como ser humano e ser social ${ }^{12}$. Contudo, os enfermeiros desenvolvem, sobretudo, ações voltadas para um corpo físico e dirigidas à saúde reprodutiva, mantendose na obscuridade outras situações vivenciadas.

Embora a mamografia e o exame clínico sejam os recomendados para o rastreamento ${ }^{1,8,11}, 90 \%$ dos casos de câncer de mama são detectados pelas próprias mulheres ${ }^{8}$. Pode-se afirmar, portanto que a promoção do autoexame seja uma estratégia eficaz para a sua detecção. Porém, o exercício hegemônico de uma clínica centrada no ato prescritivo e na produção de procedimentos, em ação substitutiva da prática que valoriza a clínica como o exercício ampliado de múltiplos profissionais, contribui para a valorização de ações voltadas para o corpo físico com o propósito de identificar anormalidades sem considerar as queixas e especificidades trazidas pelo usuário do sistema de saúde ${ }^{20}$. Embora este método de rastreio não seja recomendado como um método isolado, sua associação com o exame clínico das mamas, realizada de forma correta, pode ser uma forma eficiente na detecção desta neoplasia, sendo realizada a um baixo-custo em qualquer serviço de saúde ${ }^{21}$. 
A análise das ações desenvolvidas pelos enfermeiros assim como o motivo para e a categoria concreta do vivido identificada revelam que as práticas de cuidado dirigidas à mulher são orientadas pelo modelo biomédico ${ }^{22}$, que nem sempre considera as especificidades femininas, principalmente aquelas relacionadas à saúde sexual e reprodutiva em seu sentido mais amplo ${ }^{20,23}$.

Embora não sejam considerados mecanismos de diagnóstico precoce do câncer, o exame clínico das mamas e o autoexame consistem em estratégias de detecção precoce de anormalidades no tecido mamário. Mesmo não sendo recursos que detectem a neoplasia na fase pré-clínica, possibilitam a identificação destas anormalidades na fase clínica precoce, se tornando uma maneira eficaz na interrupção da evolução da doença a partir de tratamentos específicos ${ }^{24,25}$. No entanto, o exercício da clínica, traduzido em atos de fala/escuta, em que o diagnóstico da patologia se torna o foco central do cuidado, foi sendo ao longo do tempo substituído pelo ato prescritivo, na relação sumária entre profissional e usuário ${ }^{20}$. Há, portanto, nos serviços de saúde, e neste caso, na atenção primária de saúde, um imaginário em que assistência de qualidade é sinônimo de prescrição de medicamentos e consultas especializadas.

Vale atentar que a detecção precoce de anormalidades integra o modelo biomédico ${ }^{24}$. Embora a ação profissional dos enfermeiros entrevistados esteja fundamentada nesse modelo, as ações de detecção precoce para o câncer de mama permanecem ocultas em sua prática assistencial. Corroboram este modelo as práticas profissionais recomendadas para a atenção primária de saúde pelos manuais de assistência preconizadas pelo Ministério da Saúde ${ }^{11,18,19}$, os quais têm como referência ações profissionais voltadas para a prevenção da doença, predominantemente para o corpo físico da mulher.

Dessa forma, o enfermeiro deveria ter como um dos seus propósitos de ação profissional, a detecção precoce de anormalidades na mama, o que não vem ocorrendo. Mesmo tendo sua ação profissional fundamentada pelo modelo biomédico, esta não resulta em atitudes que visem à detecção precoce de anormalidades na mama, o enfermeiro que assiste à mulher na atenção básica se ocupa predominantemente com a prevenção de problemas no corpo gravídico.

O processo de trabalho que se observa nos dias de hoje desconhece o sujeito pleno que traz consigo além de um problema de saúde, certa subjetividade, uma história de vida, que são também determinantes do seu processo de saúde e doença ${ }^{25}$. Nesse sentido, no âmbito da detecção precoce do câncer de mama, as ações dos profissionais do enfermeiro se fazem mais do que necessárias. Cabe a estes, a partir do atendimento integral, favorecer o empoderamento da mulher quanto à sua saúde e seu corpo. Com este entendimento, tanto o profissional, nas consultas voltadas para a saúde da mulher, quanto a usuária serão capazes de detectar precocemente anormalidades na mama, que possam caracterizar eventualmente a fase inicial do câncer de mama. É fundamental a ação do enfermeiro durante as consultas na atenção à saúde da mulher para a detecção de anormalidades através do acolhimento, no exame clínico das mamas, na educação em saúde e na solicitação de exames mais complexos quando necessário. Os profissionais de saúde devem assumir a responsabilidade de participar na detecção de anormalidades na mama.

\section{Conclusão}

Esta pesquisa identificou que as ações profissionais dos enfermeiros que atendem as mulheres nas unidades básicas de saúde são, principalmente, as ações voltadas para o corpo físico gravídico da mulher. Sendo assim, o enfermeiro que assiste à mulher na atenção primária à saúde não tem como propósito da sua ação profissional, isto é, como motivos para, a detecção precoce de anormalidades na mama.

Considerando a importância da detecção precoce através do exame clínico das mamas e o autoexame das mamas, estas estratégias auxiliam a redução da morbimortalidade do câncer de mama no cenário brasileiro.

Dessa forma, é importante conhecer quem efetivamente é o sujeito do cuidado, para que a assistência possa ser oferecida de forma eficaz e satisfatória. Sugere-se que o enfermeiro assuma na sua prática assistencial a responsabilidade de participar na detecção precoce de anormalidades na mama como parte da sua atribuição e compromisso profissional com a saúde como direito.

\section{REFERÊNCIAS}

1.Instituto Nacional de Câncer José Alencar Gomes da Silva $(\mathrm{Br})$. Sistema de informação do câncer: manual preliminar para apoio à implantação. Rio de Janeiro: INCA; 2013.

2.Anderson BO, Cazap E. Breast health global iniative (BHGI) outline for program developmenting Latin America. Salud Pública de México. 2009; 51(Suppl 2):s309-15. 3.Guerra CE, Armstrong K, Sherman M. Diffusion of breast cancer risk assessment in primary care. Journal of the American Board of Family Medicine. 2009; 22:272-9. 4.Jones L, Chilton JA, Hajek RA, Iammarino N, Laufman L. Between and within: international perspectives on cancer health disparities. J Clin Oncol. 2006; 24:2204-8. 5.Instituto Nacional de Câncer José Alencar Gomes da Silva (Br). Coordenação de Prevenção e Vigilância. Estimativa 2014: incidência de câncer no Brasil. Rio de Janeiro: INCA; 2014. 
6.Ministério da Saúde (Br). Instituto Nacional do Câncer. Falando sobre câncer de mama. Rio de Janeiro: Ministério da Saúde; 2002.

7.Leavell S, Clarck EG. Medicina preventiva. São Paulo: Editora McGraw-Hill; 1976.

8.Instituto Nacional do Câncer (Br). Controle do câncer de mama. Rio de Janeiro: MS/INCA; 2004.

9.Ministério da Saúde $(\mathrm{Br})$. Secretaria de Atenção à Saúde. Política Nacional de Atenção Básica. Brasília (DF): Ministério da Saúde; 2012.

10.Conselho Federal de Enfermagem. Código de Ética dos Profissionais de Enfermagem- Resolução COFEN no 311/2007. [citado em 14 dez 2014]. Disponível em: www.coren-rj.org.br

11.Ministério da Saúde $(\mathrm{Br})$. Secretaria de Atenção à Saúde. Controle dos cânceres do colo do útero e da mama. Brasília (DF): Ministério da Saúde; 2013.

12.Ministério da Saúde $(\mathrm{Br})$. Secretaria de Atenção à Saúde. Política Nacional de Atenção Integral à Saúde da Mulher: princípios e diretrizes. Brasília (DF): Ministério da Saúde; 2007.

13.Domingos SRF. O significado da ação de provocar o aborto na adolescência: uma abordagem da fenomenologia social sob a perspectiva de mulheres [tese de doutorado]. São Paulo: Universidade de São Paulo: 2011. 14.Tocantins FR, Lima CA. Necessidades de saúde do idoso: perspectivas para a enfermagem. Rev Bras Enferm. 2009; 62:367-73.

15.Schütz A. Collected papers 1: the problem of social reality. Netherlands: Nijhoff; 1962.

16. Tocantins FR. O agir do enfermeiro em uma unidade básica de saúde: análise das necessidades e demandas [tese de doutorado]. Rio de Janeiro: Universidade Federal do Rio de Janeiro; 1993.
17.Ayres JRCM. O cuidado, os modos de ser (do) humano e as práticas de saúde. Saúde e Sociedade. 2004; 13:16-29.

18.Ministério da Saúde (Br). Secretaria de Atenção a Saúde. Departamento de Ações Programáticas Estratégicas. Pré-natal e puerpério: atenção qualificada e humanizada - manual técnico. Brasília (DF): Ministério da Saúde; 2006.

19.Secretaria Municipal de Saúde e Defesa Civil (RJ). Coordenação de Saúde da Família. Protocolos de enfermagem na atenção primária à saúde. Rio de Janeiro: Superintendência de Atenção Primária; 2012.

20.Merhy EE. A cartografia do trabalho vivo. São Paulo: Editora Hucitec; 2002.

21.Zapponi ALB, Tocantins FR, Vargens OMC. A detecção precoce do câncer de mama no contexto brasileiro - revisão integrativa. Rev enferm UERJ. 2012; 20: 386-90. 22.Camargo Junior KR, Campos EMS, BustamanteTeixeira MT, Mascarenhas MTM, Maud NM, Franco TB, et al. Avaliação da atenção básica pela ótica políticoinstitucional e da organização da atenção com ênfase na integralidade. Cad Saúde Pública. 2008; 24:58-68.

23. Gonçalves LLC, Lima AV, Brito ES, Oliveira MM, Oliveira LAR, Abud ACF et al. Mulheres portadoras de câncer de mama: conhecimento e acesso às medidas de detecção precoce. Rev enferm UERJ. 2009;17:62-7. 24.Mai V, Sullivan T, Chiarelli AM. Breast cancer screening program in Canada: successes and challenges. Salud Pública Mex. 2009; 51: 228-35.

25.Nothacker M, Duda V, Warm M, Hahn M, Degenhardt F, Madjar $\mathrm{H}$ et al. Early detection of breast cancer: benefits and risks of supplemental breast ultrasound in asymptomatic women with mammographically dense breast tissue. A systematic review. BMC Cancer. 2009; 9:335. 